\title{
Distributed Spatio-Temporal Spectrum Sensing: An Experimental Study
}

\author{
Chandrasekharan Raman*, Janani Kalyanam ${ }^{\dagger}$, Ivan Seskar*, Narayan Mandayam*, \\ *Wireless Information Network Laboratory (WINLAB) \\ Rutgers, The State University of New Jersey \\ North Brunswick, NJ 08902. \\ ${ }^{\dagger}$ Dept. of ECE, University of Wisconsin-Madison \\ Madison, WI 53706.
}

\begin{abstract}
In this experimental study, we propose simple spectrum sensing algorithms for localizing transmitters in space using frequency agile sensors that are capable of sensing only a limited bandwidth at any instant of time. We present heuristics to localize a single transmitter and multiple asynchronous sources transmitting in the same band, by applying simple triangulation techniques based on sensed power at the each sensor. We also address the problem of finding the spectral occupancy over a wide band of frequencies, using frequency agile sensors capable of sensing a limited bandwidth. We identify important practical issues in frequency agile spectrum sensing, including the observation that simple algorithms like energy detection and triangulation methods for localization, though simple, are not sufficient in "identifying" heterogeneous transmissions interfering with each other in a given location.
\end{abstract}

\section{INTRODUCTION}

With increasing demand in wireless spectrum for the growing number of applications, cognitive radios have been gaining popularity due to their ability to opportunistically use the unused white spaces in the spectrum [1]. In future wireless radio networks, cognitive radios would have to coexist with heterogeneous radios that run different static-protocols. Cognitive radio networks may operate in two modes. In a coordinated network, primary and secondary users may be controlled by a central entity, e.g., a spectrum server [2]. The secondary users are assigned time and frequency slots by the central agent depending on the activity of the primary transmitters. In an uncoordinated cognitive radio network, which is the focus of this paper, the secondary users identify white spaces to opportunistically transmit in those time and frequency slots. Observe that identifying a white space involves specifying the time, frequency and space coordinates in the spectrum. Hence, device localization and neighbor discovery techniques become essential in identifying white spaces in the spectrum. There has been recent interest in devising strategies for localizing the primary uses in a given area at a particular frequency [3], [4]. In [5], the authors propose and compare various neighbor discovery schemes for dynamic spectrum access in adhoc networks.

This work is supported in part by the NSF under grant number NeTS0434854 and by the Defense Spectrum Office (DSO) of the Defense Information Systems Agency.
In this paper, we address some of the practical issues in localization of a primary transmitter using cognitive radios that are capable of tuning their radio front-end to any desired frequency over a wide range. We refer to this property as the frequency agility property. The sensors have an additional constraint in that the sensors are capable of listening only to a limited band of frequencies at any time. Using energy detection and simple triangulation techniques, we present heuristics to localize a single transmitter in space, multiple asynchronous transmitters in space. We then address the issue of finding the spectral occupancy of a set of transmitters operating over a wide range of frequencies. In the following section, we define the problem and in section III, we explain the localization algorithms and present the results. In the last section, we conclude by identifying some of the challenges for future work in this area.

\section{Problem Statement}

We consider the scenario where there are $M$ multiple heterogeneous transmitters, e.g., IEEE 802.11 access points and bluetooth transmitters, in an indoor locality. The locations $t_{i}=\left(x_{i}, y_{i}\right), i=1, \ldots, M$ are unknown. Using a set of $N$ wireless sensor nodes, our aim is to localize the transmitters in space and find the spectral activity in a given band of interest. The wireless sensors are frequency agile and are capable of sensing a limited bandwidth (typically very much less the transmission bandwidth) at any point in time. The wireless sensors are located at known locations, $s_{j}=\left(a_{j}, b_{j}\right), j=$ $1, \ldots, N$. Under this setting, we are interested answering the following questions:

1) How do we localize interfering transmitters in space?

2) How can we find the spectral occupancy in a given band of frequencies using these sensors?

Though the above two questions are addressed in research related to device localization and neighbor discovery, the limited bandwidth sensing capability adds a new dimension to the problem. For example, consider a bluetooth transmitter that hops over a wide band according to some pseudo random sequence that is unknown to the sensors. In order to localize the bluetooth transmitter, a simple method would require sensing the power at at least three sensor locations. But when 
○

Sensor 1

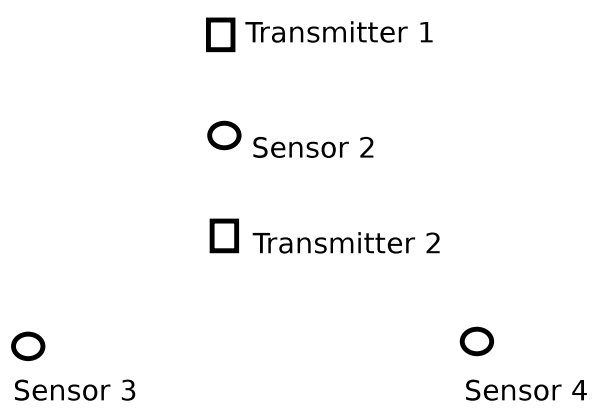

Fig. 1. Experiment set-up. The four sensors are the nodes with GNU radio interfaces. The transmitter nodes are configured as IEEE802.11b access points.

the sensors cannot sense the whole bandwidth, the sensors need to somehow measure the power in the whole bandwidth of transmission. Hence, it is required for the sensors to measure power across the whole band. To localize accurately, we may have to increase the time for localization, which is an important performance metric in cognitive radio networks. Thus, there is a need for better localization algorithms than the existing schemes because of the limited sensing bandwidth of the sensors. One of the goals of this paper is to evaluate the goodness of simple localization schemes and their limitations.

\section{EXPERIMENT SET-UP AND RESULTS}

The experiments were performed in the ORBIT radio grid at WINLAB Technology Center [6]. ORBIT, an open-access wireless testbed, is a grid of $20 \times 20$ radios, each of which is connected to a host processor. The nodes contain various radio interfaces, such as IEEE 802.11, Bluetooth, Zigbee and GNU radio interfaces. The USRP platform [7] and the GNU radio [8] together provide the radio front-end hardware and software capabilities respectively to implement a software defined radio in the ORBIT grid.

The bandwidth sensing limitation of the GNU radios comes from the fact that the digital data transfer from the RF frontend to the host computer is limited by the transfer capability of the USB 2.0 interface. This imposes a constraint on sampling rate of the ADC in the RF front-end of the USRP device. The ADC operates at a sampling rate of 64 megasamples/second. Thus, by the Nyquist sampling theorem, there is a maximum bandwidth of sensing for the GNU radio nodes. The center frequency of the RF front-end can be changed using software and this provides the frequency agility for the sensors. Thus, the nodes with GNU radio interface emulate the functionality of frequency agile sensors with limited bandwidth sensing capability. For the transmitters, some nodes in the radio grid are configured as IEEE802.11b access points (APs). The APs transmit beacons (that are nothing but IEEE802.11b packets) periodically every 100 milliseconds. In the rest of this section, we explain the heuristics considered for different experiments and briefly explain the results.

\section{A. Localizing a single transmitter in space}

We consider the scenario in Figure 1, where only Transmitter 1 is active and Transmitter 2 does not exist. The simplest experiment is localize the Transmitter 1 in space. We assume that the frequency band of transmission is known in this case. Let $W_{t}$ be the bandwidth of transmission around the center frequency $f_{0}$, i.e., the transmission in the band $\left[f_{0}-W_{t} / 2, f_{0}+W_{t} / 2\right]$. It should be noted that $W_{t}>W_{s}$, the sensing bandwidth of the sensors. We now tune all the four sensors to a center frequency $f_{s} \in\left[f_{0}-W_{t} / 2, f_{0}+W_{t} / 2\right]$. Multiple snapshots of discrete time samples are collected at each of the four sensor node locations so that the burstiness of the source is captured. The sensed power $Y_{j}(n)$ at each sensor location $j=1,2,3,4$ at time $n$ is calculated by evaluating the FFT of the discrete time samples and integrating the FFT over the sensing bandwidth $W_{s}$. The measurements are taken with no coordination among the sensors.

Figure 2 shows the sensed power $Y_{j}(n)$ at different time instances $n$ at each of the four sensors, $j=1,2,3,4$. After applying a power threshold to remove uncertainties due to noise, we find the histogram of the sensed power in Figure 3. The histogram of the sensed power gives the distribution of the sensed power at each of the four sensors. We now use simple triangulation techniques to localize the transmitter. The results obtained from the experiment allow us to localize the transmitter within an error of 15 feet.

\section{B. Localizing multiple asynchronous transmitters in space}

When we have two transmitters transmitting in the same band, uncoordinated measurements with the sensors leads to inconclusive results as we may not be able to distinguish the transmitters from the sensed power. Since we require some way to distinguish the transmission from two different sources, we synchronously sense the power at each sensor from the transmitter. By exploiting the asynchronous transmissions from the sources, we localize multiple transmitters in space. Towards this end, we consider the scenario in Figure 1 where both transmitters are transmitting in the same channel. Assuming that we know the band of frequencies of transmissions, we follow the same steps as described above to localize a single transmitter. The histogram reveals a bimodal distribution of power at the sensor locations. The results obtained from the experiment allow us to localize both transmitters within an error of $13-16$ feet.

\section{Finding the spectral occupancy over a band of frequencies}

In the previous two experiments, we assumed that we know the frequencies of transmission of the sources. In reality, however, the band of frequencies in which the source is active is unknown. In such cases, before we localize the transmitters in space, we need to localize the band of frequencies in which the sources are active. In a broader sense, finding the spectral occupancy in a given band of frequencies using frequency agile sensors capable of sensing a limited bandwidth is a challenge. A simple way of finding the spectral occupancy of 

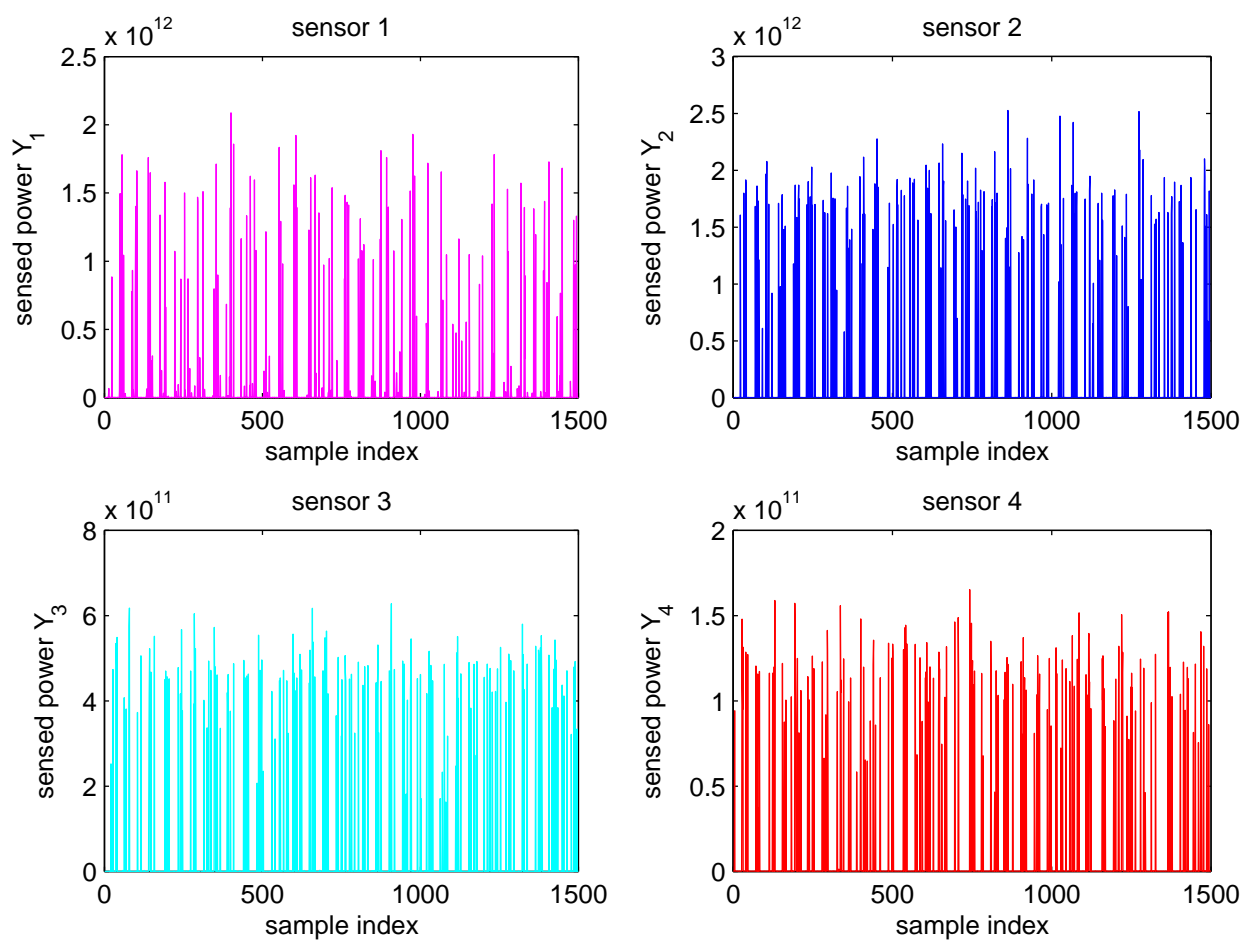

Fig. 2. Sensed power over $W_{s}=4 \mathrm{MHz}$ bandwidth in the case of a single transmitter.
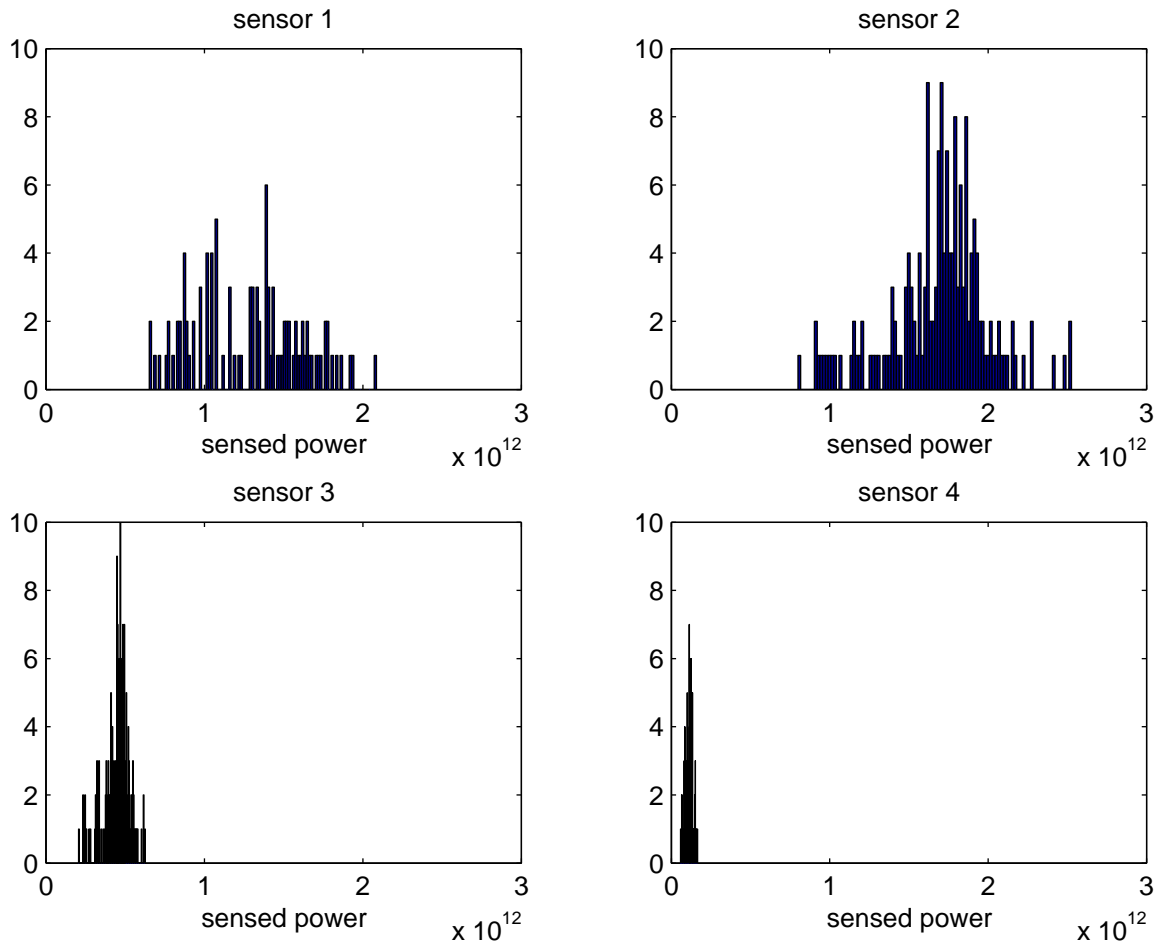

Fig. 3. Histogram of the sensed power at the four sensors after setting a threshold. 
Sensor 1

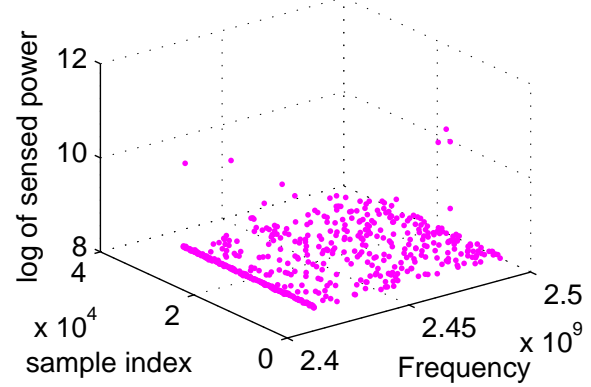

Sensor 3

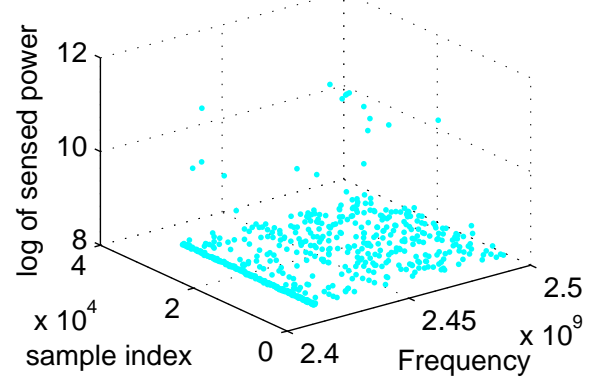

Sensor 2

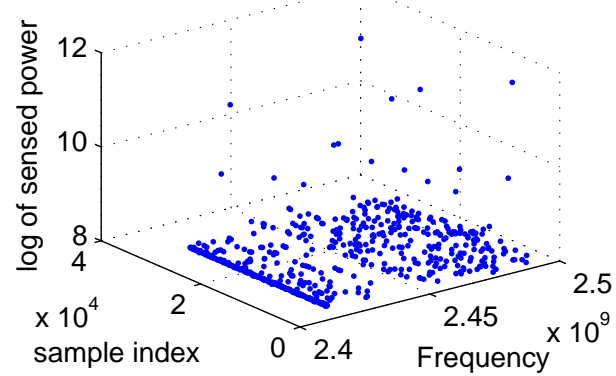

Sensor 4

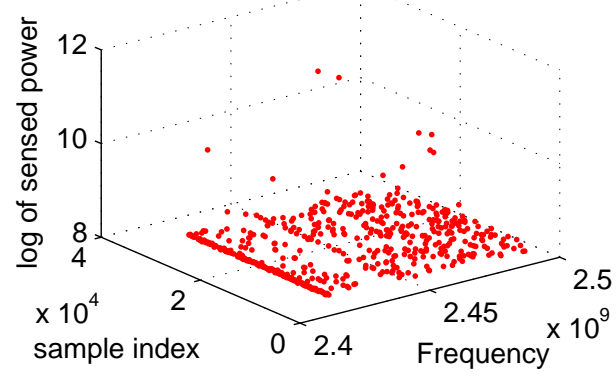

Fig. 4. Three dimensional plot showing the spectral activity as seen by each of the four sensors in the $2.41-2.49 \mathrm{GHz}$ band over the sensing period.
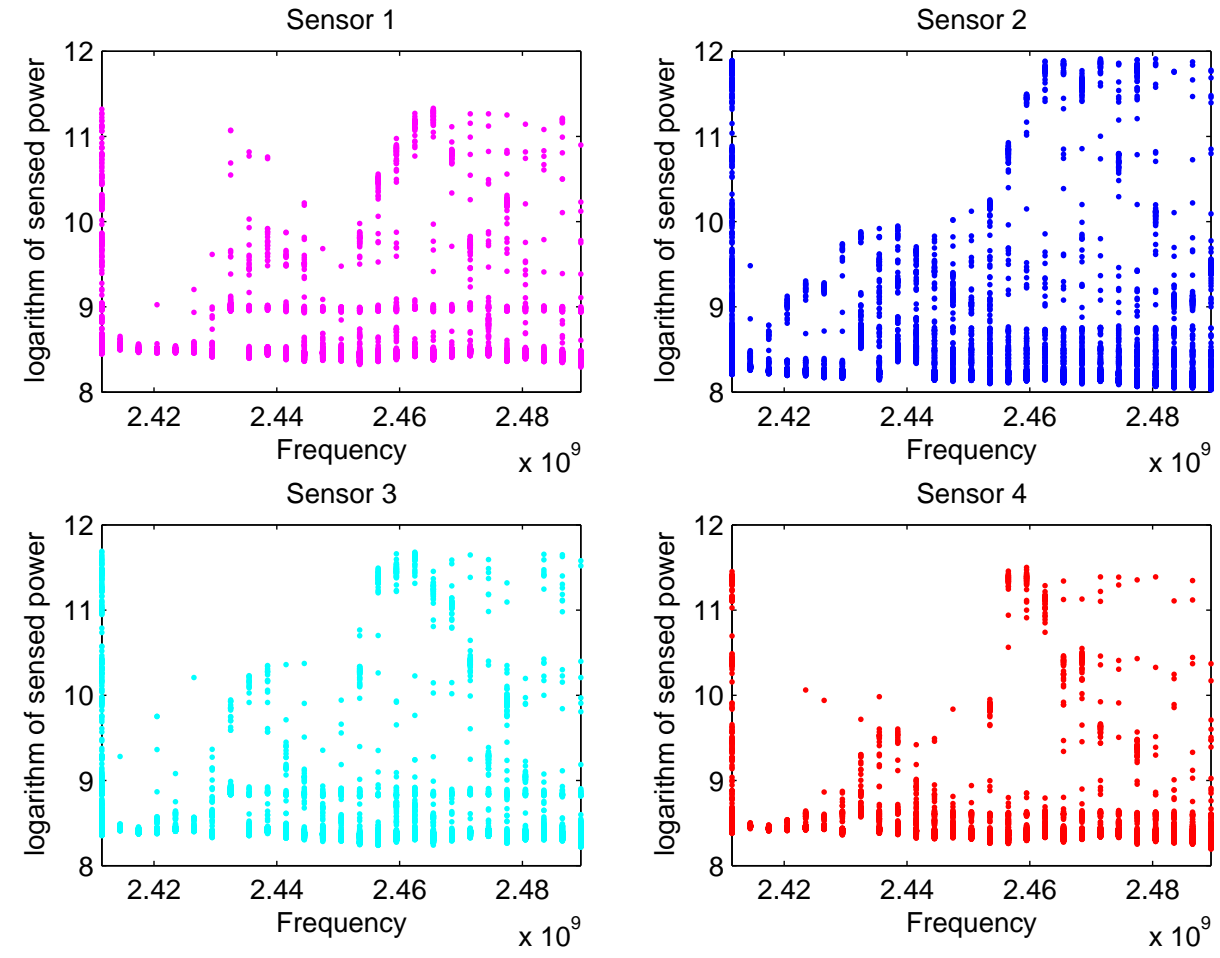

Fig. 5. Two dimensional projection of the spectral activity in Figure 4. A point in the graph denotes the logarithm of the sensed power around the center frequency at some point in time during the sensing interval. 
a given band using sensor that can sense only a limited bandwidth is to continuously sweep the whole band of frequencies using all the sensors. But if the sources are bursty, the sensors may miss the activity of the band of interest. This may lead to very long sensing time and thus may not be an effective solution. Splitting the whole band into several sub-bands and employing different sensors to sense different bands may require more sensors to improve the accuracy of localization. In this work, we resort to a randomized algorithm [9] to address this issue.

Let us now consider the set up in Figure 1 where Transmitter 2 is the only active transmitter. Let $W_{t}$ be the bandwidth of transmission around the unknown center frequency $f_{0}$, i.e., the transmission in the band $\left[f_{0}-W_{t} / 2, f_{0}+W_{t} / 2\right]$. It is required to identify the band of transmission over a range of frequencies $W\left(>W_{t}\right)$ of frequencies. We now allow each sensor $j$ to randomly jump to a center frequency $f_{j}(n) \in$ $W$. The random center frequencies are chosen independently and uniformly across all the sensors. In our experiment, we are interested in the spectral activity in the band $2.41 \mathrm{GHz}$ to $2.49 \mathrm{GHz}$, i.e., $W=80 \mathrm{MHz}$. Figure 4 shows the three dimensional plot of spectral occupancy as seen by the four sensors in the $2.41-2.49 \mathrm{GHz}$ band over a period of time sufficient to capture the burstiness in the transmitting source. In our experiments, we collect samples for around $300-400$ seconds. Figure 5 shows the two dimensional projection of the three dimensional plot with the time axis suppressed. We plot the envelope of the logarithm of sensed power at the four sensor locations in Figure 6. Notice that there is some activity in the channel 11 of the IEEE $802.11 \mathrm{~b}$ band $(2.46 \mathrm{GHz})$.

\section{Discussion AND CONClusion}

In the previous section, we proposed simple heuristics to localize a single transmitter in space, multiple asynchronous interfering transmitters in space and to find the spectral occupancy over a wide band using frequency agile sensors capable of sensing a limited bandwidth. Although these simple algorithms work very well to localize transmitters in space and frequency, there are lot of limitations for these algorithms. We discuss some of the limitations and open issues in this section.

- When there are multiple sources transmitting in the same band, energy detection techniques are insufficient to localize the transmitters when they are simultaneously transmitting. This is because each transmitter contributes different unknown proportions of power at the sensor nodes. Simple methods based on received power measurements are inconclusive. Isolating each transmitter based on the transmitter's signature are essential to localize the transmitters in this scenario. This would require decoding the packet in the case of IEEE 802.11 protocol transmissions.

- When localizing multiple interfering transmitters, synchronous sensing proved helpful. When the sensors are synchronized, the granularity of message exchange is an important parameter to consider. Synchronization overhead can be very expensive and may not be affordable

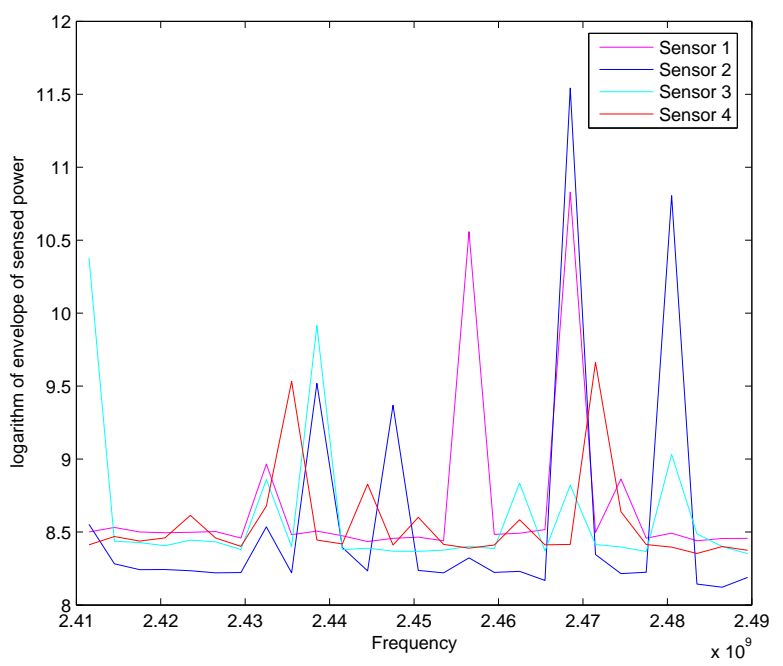

Fig. 6. Envelope of the logarithm of sensed power over time in the 2.41 $\mathrm{GHz}$ to $2.49 \mathrm{GHz}$ band. We observe activity in the channel 11 of the IEEE $802.11 \mathrm{~b}$ band.

in certain network applications. Coordination between sensors to establish synchronization can be a practical challenge.

- There exists a trade-off between the cost and complexity of the sensors. When we require complex signal processing algorithms in the cognitive radio sensors, the cost of the devices increases. Thus, there is a need for advanced signal processing and detection algorithms which can be implemented at low cost.

- The time taken for localization directly impacts the time to find the white spaces in the spectrum. In highly dynamic networks, where white spaces do not last longer, time to localize needs to be lower so that secondary users can make better use of the white spaces. Hence algorithms that take lower time to localize are preferable in cognitive radio networks.

\section{REFERENCES}

[1] D. Cabric, S. Mishra, D. Willkomm, R. Brodersen, and A. Wolisz, "A cognitive radio approach for usage of virtual unlicensed spectrum," in Proc. of the 14th IST Mobile and Wireless Comm. Summit, June 2005. Baltimore, MD.

[2] N. Mandayam, "Cognitive algorithms and architectures for open access to spectrum," Conf. on the Economics, Technology and Policy of Unlicensed Spectrum, May 2005. East Lansing, MI.

[3] J. Nelson, M. Hazen, and M. Gupta, "Global optimization for multiple transmitter localization," in Proc. MILCOM, 2006. Washington DC.

[4] J. Nelson and M. Gupta, "An EM technique for multiple transmitter localization," in Proc. CISS, 2007. Baltimore, MD.

[5] K. Balachandran and J. H. Kang, "Neighbor discovery with dynamic spectrum access in adhoc networks," in Proc. of Vehicular Technology Conf., May 2006. Melbourne.

[6] "ORBIT: Open-Access Research Testbed for Next-Generation Wireless Networks." http://www.orbit-lab.org/.

[7] "The Universal Software Radio Peripheral." http://www.ettus.com/.

[8] "GNU Radio: the gnu software radio." www.gnu.org/software/gnuradio.

[9] R. Motwani and P. Raghavan, Randomized Algorithms. Cambridge University Press, NY, 1995. 